\title{
Colesteatoma do Canal Auditivo Externo: Caso Atípico de Apresentação Bilateral
}

\section{Bilateral External Auditory Canal Cholesteatoma: Case Report}

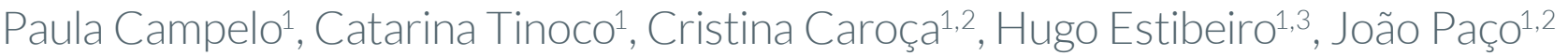

\section{RESUMO}

INTRODUÇÃO: O colesteatoma do canal auditivo externo é raro e muitas vezes assintomático apesar da insidiosa destruição óssea que vai causando no canal auditivo externo e estruturas vizinhas. Relatos de doença bilateral são episódicos e de causa idiopática. A keratosis obturans é o principal diagnóstico diferencial.

CASO CLínICO: Criança, 13 anos, com colesteatoma bilateral do canal auditivo externo, com invasão da mastoide e erosão da cadeia ossicular no ouvido esquerdo. A doente apresentava quadro arrastado de otalgia bilateral, com otorreia esporádica e hipoacusia progressiva à esquerda. Após vários procedimentos cirúrgicos para erradicação da doença, mantém-se em vigilância anual sem recidiva aos 4 anos.

CONCLUSÃO: Este caso realça a necessidade do reconhecimento precoce desta entidade pouco frequente, mas que pode cursar com lesões extensas e até irreversíveis. O tratamento cirúrgico é curativo, mas a vigilância regular é mandatória.

PALAVRAS-CHAVE: Colesteatoma; Doenças do Ouvido; Meato Acústico Externo 


\begin{abstract}
INTRODUCTION: Cholesteatoma of the external auditory canal is rare and often asymptomatic despite the insidious bone destruction that it causes in the external auditory canal and surrounding structures. Reports of bilateral disease are episodic and usually idiopathic. Keratosis obturans is the main differential diagnosis.
\end{abstract}

CLINICAL CASE: A 13-year-old child diagnosed with bilateral cholesteatoma of the external auditory canal, associated with mastoid invasion and erosion of the ossicular chain in the left ear. The patient presented with bilateral otalgia, sporadic otorrhea and progressive hearing loss on the left ear. After several surgical procedures to eradicate the disease, the patient remains under annual surveillance without relapse after 4 years.

CONCLUSION: This case emphasizes the need for early recognition of this uncommon entity that can occur with extensive and even irreversible lesions. Surgical treatment is curative but regular surveillance is mandatory.

KEYWORDS: Cholesteatoma; Ear Canal; Ear Diseases

\section{INTRODUÇÃO}

O colesteatoma do canal auditivo externo (CCAE) é uma entidade rara cuja fisiopatologia ainda não está totalmente esclarecida. Ao contrário do (comum) colesteatoma do ouvido médio, o CCAE caracteriza-se numa fase inicial por uma hiperplasia epitelial com infeção e periostite secundária no canal auditivo externo (CAE) e posteriormente pela erosão óssea do CAE que resulta na acumulação de debris epidérmicos e espículas ósseas. ${ }^{1}$

Relatos de doença bilateral são episódicos e levantam questões, principalmente no diagnóstico diferencial com a keratosis obturans (KO).1-3 Neste trabalho apresenta-se um caso invulgar de CCAE bilateral com invasão do ouvido médio e erosão da cadeia ossicular à esquerda.

\section{CASO CLÍNICO}

Criança do género feminino, 13 anos, saudável, sem patologia otológica prévia que vem à consulta por quadro arrastado de otalgia bilateral ligeira, associado a queixas de otorreia esporádica e hipoacusia progressiva, nos últimos meses, mais marcada à esquerda.

Ao exame objetivo constatou-se a presença de otorreia purulenta e debris epidérmicos que ocluíam por completo ambos os CAEs, impedindo a visualização direta do tímpano. O restante exame otorrinolaringológico (ORL) não apresentava alterações de relevo.

O audiograma tonal confirmou a presença de hipoacusia de condução ligeira à direita e moderada à esquerda, segundo a classificação do Bureau International d'Audiophonologic (BIAP). ${ }^{4}$ A tomografia computorizada (TC) documentou um alargamento generalizado de ambos os CAEs à custa de erosão óssea, nomeadamente ao redor do annulus timpânico. À direita, observava-se o CAE preenchido por uma massa de densidade homogénea, tipo tecido de partes moles, sem sinais de envolvimento do ouvido médio; à esquerda, constatava-se a presença de uma massa de semelhante densidade com extensão ao epitímpano e mesotímpano externo através da pars flácida que condicionava erosão extensa da bigorna (Fig. 1).

Num primeiro tempo, a doente foi submetida a mastoidectomia radical esquerda para erradicação da doença. No intraoperatório constatou-se a presença de queratina com erosão óssea do CAE, membrana timpânica e erosão quase total do martelo e bigorna, não tendo sido realizado tempo funcional. A anatomia patológica revelou tecido escamoso queratinizante compatível com colesteatoma.

Após 5 meses, num segundo tempo cirúrgico, procedeu-se à remoção do tecido queratinizante do CAE direito e realização de canaloplastia e meatoplastia por via retroauricular com revestimento das paredes ósseas com enxerto livre de pele. A membrana timpânica encontrava-se preservada.

O pós-operatório da última cirurgia complicou com uma infeção tardia, observando-se encerramento do CAE por tecido fibrótico, em fundo de saco, o qual motivou nova intervenção cirúrgica. A baixa tolerância e colaboração da doente para os cuidados de higiene otológica poderão ter contribuído para o desenrolar da situação.

Desde a última intervenção cirúrgica, a doente mantém-se em vigilância clínica anual, sem sinais de recidiva. A recuperação da hipoacusia à direita foi total. No entanto, devido à hipoacusia de condução sequelar no ouvido esquerdo a doente foi proposta para colocação de implante osteointegrado (BAHA ${ }^{\circledR}$ ) à esquerda que tem utilizado de forma satisfatória. 


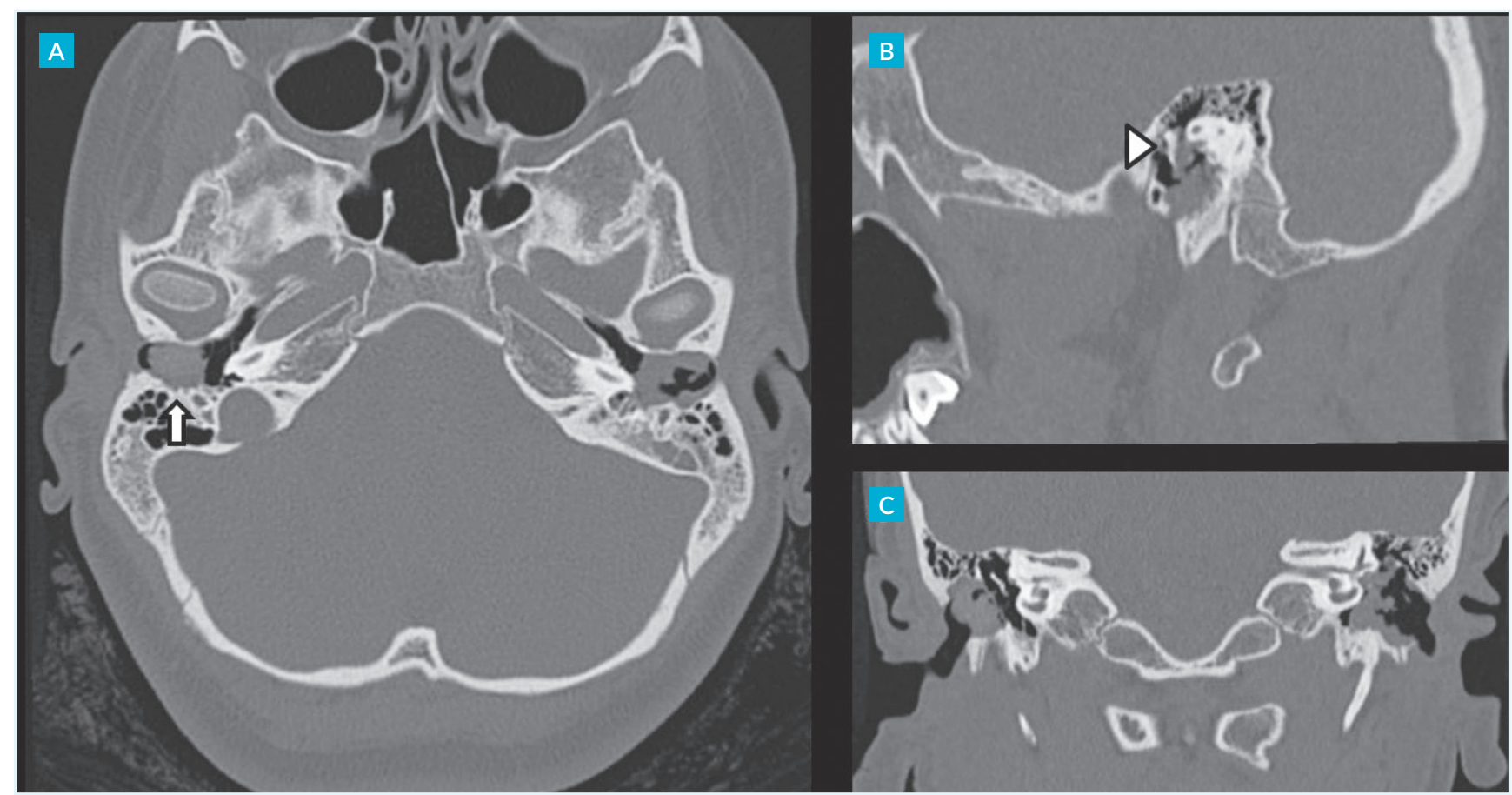

FIGURA 1. TC pré-operatório do osso temporal, (A) corte axial, (B) corte sagital do ouvido esquerdo (C) corte coronal. Ouvido direito: Lesão com densidade de tecidos de partes moles centrada na porção óssea do CAE, condicionando erosão da parede posterior (seta), sem invasão do ouvido médio. Ouvido esquerdo: Lesão com densidade de tecidos de partes moles que preenche o 1/3 interno do CAE, condicionando erosão das paredes ósseas com extensão ao ouvido médio e erosão ossicular, nomeadamente da bigorna (cabeça de seta).

\section{DISCUSSÃO}

O diagnóstico do CCAE baseia-se na história clínica e exame físico. Caracteristicamente, e quando sintomático, o CCAE apresenta-se com otorreia escassa, unilateral acompanhada de otalgia pouco intensa. Na maioria dos casos a hipoacusia não está presente. Tipicamente a lesão ocorre no pavimento do CAE, mas outras localizações foram já descritas. ${ }^{1}$ Pela sua clínica pouco específica, o CCAE pode ser difícil de diferenciar de outras patologias, em especial da KO, já que ambas se caracterizam pela acumulação de queratina no CAE. ${ }^{5}$

Caracteristicamente, o CCAE é descrito como uma patologia unilateral de adultos/idosos de caráter arrastado e associada a desconforto e otorreia, ao contrário da KO presente de forma bilateral, no adulto jovem, associada a dor forte, podendo estar associada a bronquiectasias ou sinusite. ${ }^{2}$

O padrão de invasão óssea é o principal elemento distintivo entre as duas. ${ }^{5}$ O CCAE é caracterizado pela erosão do osso, normalmente localizada na região póstero-inferior do CAE, com a presença de espículas ósseas, necrose óssea e padrão desorganizado de deposição das lamelas de queratina. Uma perda focal do epitélio com exposição óssea parece também estar apenas presente nos casos de CCAE. ${ }^{\circ}$ A KO, por sua vez, apresenta um marcado alargamento circunferencial do CAE decorrente da expansão óssea e não erosão. ${ }^{5}$ A Anatomia Pa- tológica, além de excluir outros diagnósticos, pode contribuir para o diagnóstico diferencial entre estas duas entidades. Nos estudos de patologia publicados, na KO não se observava o padrão erosivo do CCAE mas a presença de marcadores inflamatórios e da dilatação vascular no tecido subepitelial; a organização das lamelas de queratina era também diferente, mais organizada, de forma circunferencial e centrípeta. ${ }^{6}$

No caso descrito, o diagnóstico diferencial entre CCAE e KO coloca várias questões. Pela idade pediátrica, apresentação bilateral e alargamento do CAE, a KO seria o diagnóstico mais provável. No entanto, a erosão óssea, a presença de espículas ósseas e a perda focal de epitélio são características descritas por Persaud ${ }^{6}$ como distintivas do CCAE em relação à KO. Considera-se, assim, que o caso descrito constitui uma apresentação de CCAE que, apesar de rara não é única. Há relatos tanto de doença bilateral ${ }^{6,7}$ como de apresentação em idade pediátrica. ${ }^{5}$ A invasão da cavidade timpânica e destruição ossicular no ouvido esquerdo, com consequente surdez de condução é outro fator a favor do CCAE. Outros autores $^{2,7}$ descrevem extensão do CCAE às estruturas vizinhas, nomeadamente à mastoide, o local mais frequentemente envolvido após destruição da parede posterior do canal. Nestes casos a requisição da TC apresenta particular interesse nomeadamente na avaliação da extensão ao nível da cavidade timpânica, integridade no nervo facial, tegmen tympani e células mastóideas. 


\section{CONCLUSÃO}

Apesar de ser uma patologia rara, principalmente em idade pediátrica, o CCAE deve ser tido em conta no diagnóstico diferencial de lesões do CAE, mesmo em casos bilaterais já que, pela sua evolução insidiosa, o CCAE pode cursar com lesões extensas e até irreversíveis.

\section{APRESENTAÇÕES/PRESENTATIONS:}

Este trabalho foi apresentado, em formato de póster, no $61^{\circ}$ Congresso da Sociedade Portuguesa de Otorrinolaringologia e Cirurgia Cérvico-Facial (SPORL).

CONFLITOS DE INTERESSE: Os autores declaram não ter qualquer conflito de interesse na realização do presente trabalho.

FONTES DE FINANCIAMENTO: Não houve qualquer fonte de financiamento na realização do presente trabalho.

CONFIDENCIALIDADE DOS DADOS: Os autores declaram ter seguido os protocolos da sua instituição acerca da publicação dos dados de doentes.

PROTEÇÃO DE PESSOAS E ANIMAIS: Os autores declaram que os procedimentos seguidos na elaboração do presente trabalho estão em conformidade com as normas das comissões de investigação clínica e de ética, bem como da declaração de Helsínquia e da Associação Médica Mundial.

CONFLICTS OF INTEREST: The authors declare that they have no conflicts of interest.

FINANCIAL SUPPORT: This work has not received any contribution, grant or scholarship.

CONFIDENTIALITY OF DATA: The authors declare that they have followed the protocols of their work center on the publication of data from patients.

PROTECTION OF HUMAN AND ANIMAL SUBJECTS: The authors declare that the procedures followed were in accordance with the regulations of the relevant clinical research ethics committee and with those of the Code of Ethics of the World Medical Association (Declaration of Helsinki).

\section{REFERÊNCIAS}

1. Dubach P, Mantokoudis G, Caversaccio M. Ear canal cholesteatoma: meta-analysis of clinical characteristics with update on classification, staging and treatment. Curr Opin Otolaryngol Head Neck Surg. 2010;18:369-76.

2. Park SY, Jung YH, Oh J-H. Clinical characteristics of keratosis obturans and external auditory canal cholesteatoma. Otolaryngol Head Neck Surg. 2015;152:326-30.

3. Owen HH, Rosborg J, Gaihede M. Cholesteatoma of the external ear canal: etiological factors, symptoms and clinical findings in a series of 48 cases. BMC Ear Nose Throat Disord. 2006:6:16.
4. International Bureau for Audiophonology. BIAP Recommendation 02/1: Audiometric Classification of Hearing Impairments [Internet]. 1996 [cited 2017 Mar 19]. Available from: https://www.biap.org/de/recommandations/recommendations/tc-02-classification/213-rec-02-1-en-audiometric-classification-of-hearing-impairments/file.

5. Yoon Y, Park CH, Kim E, Park YH. Clinical characteristics of external auditory canal cholesteatoma in children. Otolaryngol Head Neck Surg. 2008;139(5):661-4.

6. Persaud R, Hajioff D, Thevasagayam MS, Wareing MJ, Wright A. Keratosis obturans and external ear canal cholesteatoma: how and why we should distinguish between these conditions. Clin Otolaryngol Allied Sci. 2004;29:577-81.

7. Heilbrun ME, Salzman KL, Glastonbury CM, Harnsberger HR, Kennedy RJ, Shelton C. External auditory canal cholesteatoma: Clinical and imaging spectrum. AJNR Am J Neuroradiol. 2003;24:751-6. 